\title{
PICOSECOND TIME RESOLVED ABSORPTION EXPERIMENTS OF IODINE IN COMPLEXING SOLVENTS
}

\author{
MARCEL BESNARD,$\dagger$ NATHALIE DEL CAMPO,$\uparrow$ \\ PHILIPPE FORNIER DE VIOLET $\ddagger$ and CLAUDE RULLIERE
$\dagger$ Laboratoire de Spectroscopie Moléculaire et Cristalline
U.R.A.124-C.N.R.S. U.R.A.348-C.N.R.S. \\ $\ddagger$ Laboratoire de Photochimie et Photophysique Moléculaire, \\ $\S$ Centre de Physique Moléculaire Optique et Hertzienne, \\ U.R.A.283-C.N.R.S. \\ Université de Bordeaux I, 351, Cours de la Libération, \\ 33405 Talence Cedex (France)
}

(Received in final form 2 May 1991)

\begin{abstract}
Transient absorption spectra of $\mathrm{I}_{2}$ diluted in "inert" $\left(\mathrm{CCl}_{4}, n\right.$-heptane) solvents and electron donor solvents of increasing complexing strength $\left(\mathrm{CS}_{2}\right.$, benzene, dioxane, pyridine) have been measured (in the spectral range $400-800 \mathrm{~nm}$ ) at different times (from 20 ps to $1 \mathrm{~ns}$ ) after excitation using 25 ps pulses at $\lambda=532 \mathrm{~nm}$. In inert solvents, the time evolution of the transient spectra are consistent with the model recently proposed by Harris et al. ${ }^{1}$

Such a behaviour is in marked contrast with the experimental observation in strongly complexing solvents (pyridine). This is interpreted as resulting from a competition between geminate recombination and formation of a complex between the solvent and atomic iodine $\left(\mathrm{I}^{\bullet}\right)$ formed by the photodissociation process. In intermediate complexing solvents these two processes seem to coexist.
\end{abstract}

KEY WORDS: Iodine complex, picosecond, transient absorption.

\section{INTRODUCTION}

The recombination of iodine-atoms following photodissociation of iodine molecule has been extensively studied over the past thirty years. ${ }^{1,10}$ Previous work in 1974, using "picosecond" spectroscopy, reported a fast process (the characteristic time scale of which was of the order of $100 \mathrm{ps)} \mathrm{interpreted} \mathrm{as} \mathrm{mostly} \mathrm{due} \mathrm{to} \mathrm{the}$ recombination of dissociated pair of iodine atoms recombining to form molecular iodine (geminate recombination). ${ }^{10}$

However, on theoretical and experimental basis, it has been shown that the vibrational relaxation of the nascent molecular iodine may also explain as well these previous time-resolved experimental results leading to a controversial interpretation of primary steps following photodissociation. In particular, the vibrational relaxation may also occur on a time scale of the order of $100 \mathrm{ps}^{7}$

More recently, time-resolved spectroscopic techniques, on a picosecond timescale, have provided new-insight on the dynamics of such a system in non- 
complexing solvents. ${ }^{1-4}$ Using a very high time-resolution (1 ps) and different pump and probe discrete wavelengths, Harris et al. ${ }^{1-4}$ were able to develop a model which provides a complete understanding of the excited iodine relaxation pathways. Moreover, this model was able to take into account in a consistent and revisited way all the latest observations. ${ }^{1-4}$

According to this model, in non-complexing solvents, just after the excitation step in the $B$ excited state manifold, the predissociation process is completed in less than 1 ps. The geminate recombination then occurs rapidly in less than $2 \mathrm{ps}$, leading to excited $\mathrm{I}_{2}{ }^{*}$ molecules. By vibrational relaxation, these molecules may populate the lower energy A, $\mathrm{A}^{\prime}$ excited states and the vibrationally excited levels of the $X$ ground state (see Figure 1 ). Then the population of the $A, A^{\prime}$ states decays more slowly than that of the vibrational ground state.

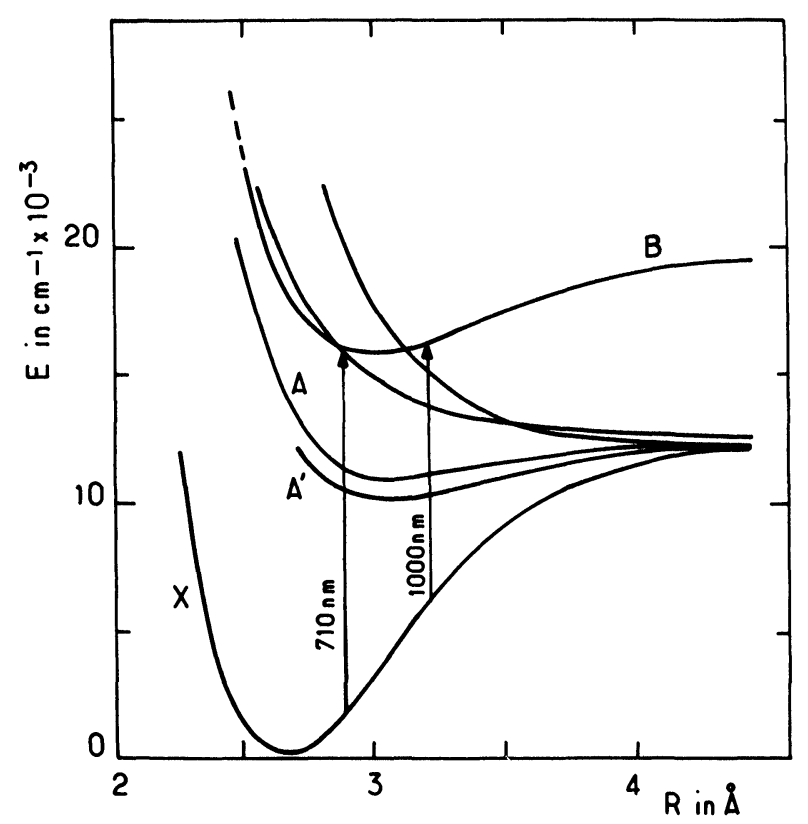

Figure 1 Electronic potential surfaces of $I_{2}$ relevant to the discussion in this paper.

However, practically all these recent studies have been performed in noncomplexing solvents and very few recent experiments have dealt with iodine molecules involved in charge-transfer complexes, i.e. in complexing solvents. As far as we know, "picosecond" experiments on systems involving iodine as an electron acceptor in electron donor solvents have been scarcely reported. ${ }^{9}$ Furthermore, such experiments were performed before the Harris interpretation.

At that time it was believed that the recombination process occurred on a 100 ps time scale and it was assumed that the complexation of iodine atoms with the solvent may compete with the recombination. According to the Harris model, in which the 
recombination occurs on a 2 ps time scale, ${ }^{3}$ the competition between this process and the complexation still remains an open question.

The aim of this paper is thus to provide a new insight on this problem. For this purpose we put emphasis in our study on the choice of the solvents and on the experimental techniques. We have selected systems in which iodine is diluted in electron donor solvents of increasing complexing strength and ranging from "inert" ( $n$-heptane, $\left.\mathrm{CCl}_{4}\right)$ to intermediate $\left(\mathrm{CS}_{2}\right.$, benzene, dioxane) and to strong donor character (pyridine). ${ }^{11}$

Most of the previous studies were performed by probing transient species at a single wavelength and thus full spectra were not available. In contrast, we use here an experimental set-up which allows us not only to measure the whole absorption spectra of transient species present in the solution at different times (from 0 to $3 \mathrm{~ns}$ ) after the excitation step, but also to probe the dynamics from measurements at single wavelength. Such a system should allow us to observe the "finger-print" in the range $400-900 \mathrm{~nm}$ of any eventual new species present in the solution.

\section{EXPERIMENTS}

All the solvents (H.P.L.C. grade) and iodine (>99\%) were purchased from Aldrich and used without further purification. The iodine concentration ranges from $7 \times 10^{-3} \mathrm{M} / \ell$ to $2 \times 10^{-2} \mathrm{M} / \ell$ depending on the solvent used.

Transient absorption experiments were performed using an experimental set-up previously described. ${ }^{12}$ The excitation source is provided by the second harmonic $(532 \mathrm{~nm}$ ) of a mode-locked Nd/YAG laser delivering pulses of $25 \mathrm{ps}$ duration. The probe source is a continuum of light extending from 400 to $900 \mathrm{~nm}$ generated by focusing the laser beam at $1.06 \mu \mathrm{m}$ into a $\mathrm{D}_{2} \mathrm{O}$ cell. The time resolution is about $10 \mathrm{ps}$ using a deconvolution procedure. A sample cell with quartz windows $(1 \mathrm{~cm}$ optical pathway) was used.

\section{EXPERIMENTAL RESULTS}

\section{(a) Inert Solvents ( $\mathrm{n}$-heptane, $\mathrm{CCl}_{4}$ )}

Figure 2 displays some transient spectra of excited iodine solutions at different times after the excitation and kinetics data plotted at different wavelengths of the transient spectra. The experimental results may be summarized as follows. We observed transient spectra belonging to two main spectral domains: A broad transient absorption in the wavelength range (580-800 $\mathrm{nm}$ ) and a rather weak and narrow absorption band near $430 \mathrm{~nm}$ (it is noteworthy that the experimental sensitivity drastically drops near $430 \mathrm{~nm}$ and that this band may possibly extend to lower wavelengths).

The spectral shape of the broad band $(580-800 \mathrm{~nm})$ changes drastically as a function of time for delay times under $200 \mathrm{ps}$. We observe that on the low energy side $(700-800 \mathrm{~nm})$ the transient spectrum has a faster rise and decay time than the high 

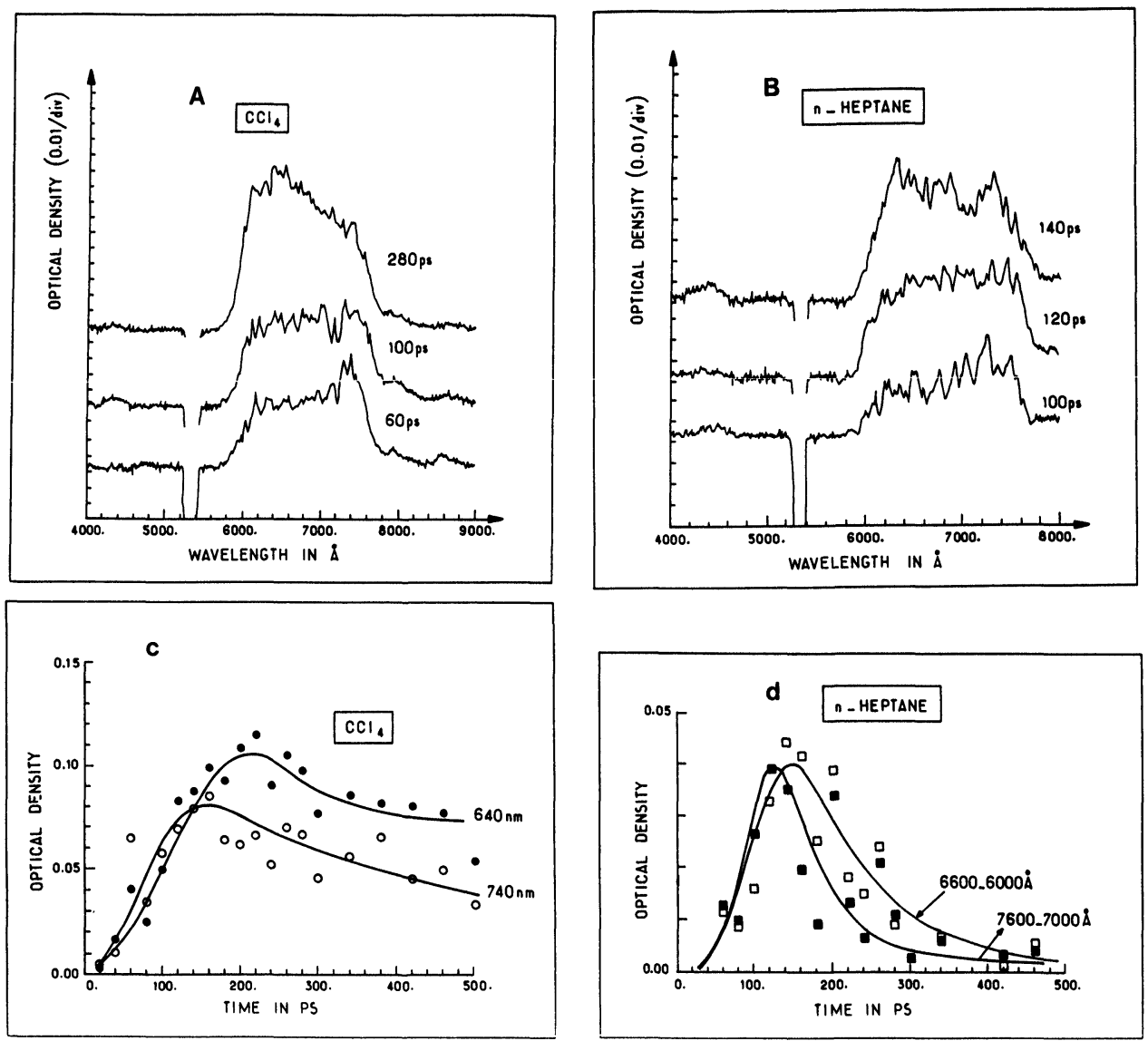

Figure 2 Transient absorption spectra recorded at increasing delay times after the excitation pulse for iodine diluted in non interacting solvents: (a) $-\mathrm{CCl}_{4}$; (b) $n$-heptane. The time evolution of the transient optical density measured at different wavelengths from previous spectra is also reported for these systems (c, d).

energy side $(580-700 \mathrm{~nm})$. This behaviour corresponds to the vibrational relaxation of $\mathrm{I}_{2}{ }^{*}$ molecule in the $X$ state as observed by Harris et al. At longer times ( $\left.\geqq 200 \mathrm{ps}\right)$, the shape remains constant and the corresponding transient decays with a lifetime of the order of $100 \mathrm{ps}$ in $n$-heptane. In $\mathrm{CCl}_{4}$, depending on the wavelength, the decay may be mono-exponential or bi-exponentional with a short component of the order of 300 ps and a long component larger than $1 \mathrm{~ns}$. (our experimental system does not allow to measure life time greater than $1 \mathrm{~ns}$ with a great accuracy). Finally the transient absorption spectra observed at $t>290$ ps looks quite similar in the two solvents. According to the Harris model, ${ }^{1}$ the spectra, obtained at times longer than $200 \mathrm{ps}$, correspond to the absorption of $I_{2}$ molecules trapped in the $A, A^{\prime}$ excited states. Finally the weak band observed at $430 \mathrm{~nm}$, which has been also previously reported, ${ }^{1,8}$ may be attributed to the virtual transition starting from the inner turning point of the $X$ potential state. ${ }^{1}$

From these results in inert solvents, we have thus obtained the absorption spectra 
of the trapped $\mathrm{I}_{2}{ }^{*}$ molecules in $\mathrm{A}, \mathrm{A}^{\prime}$ states. Therefore, using complexing solvents it should be possible, from these spectra, to obtain information about a possible competition between population of this state and population of complexes with the solvent.

\section{(b) Weakly Complexing Solvents ( $\mathrm{CS}_{2}$, Benzene, Dioxane)}

At short times ( $<150 \mathrm{ps)}$ (Figure 3), practically the same behaviour is observed as in inert solvents: the spectral shape evolves with faster kinetics (rise and decay-time) on the low energy side than on the high energy side. This is due again to vibrational relaxation in the $X$ state. At time longer than $150 \mathrm{ps,} \mathrm{the} \mathrm{spectral} \mathrm{shape} \mathrm{of} \mathrm{the}$ transient does not change significantly. But these spectra differ from those attributed to the $\mathrm{A}, \mathrm{A}^{\prime}$ excited state in inert solvents and they look like a surperposition of the $\mathrm{A}, \mathrm{A}^{\prime}$ excited states spectrum observed in inert solvent and of a new transient spectra in the lower energy side. This additional transient absorption may be due to a complexation with the solvent.

Incidentally, the presence of the weak band near $430 \mathrm{~nm}$ observed in inert solvents is also observed for benzene and $\mathrm{CS}_{2}$. In $\mathrm{CS}_{2}$ this is more apparent than in other solvents and its decay time is close to that of the main transient.

\section{(c) Strongly Complexing Solvent (Pyridine)}

Results obtained with this solvent are displayed on Figure 4 . We do not observe in the 590-800 $\mathrm{nm}$ range any band shape change as a function of time for the broad transient spectrum, which decays bi-exponentially with characteristic times equal to $300 \mathrm{ps}$ and larger than $1 \mathrm{~ns}$. The observed spectra are now quite different from those obtained at similar delay times $(t>200 \mathrm{ps})$ in inert solvents. They are in the same spectral region as the shoulder observed in inert solvents and are now attributed to the formation of a complex with the solvent. We have repeated such experiments by varying the excitation wavelength, from the second harmonic $(532 \mathrm{~nm})$ to the third harmonic $(355 \mathrm{~nm})$ of the pump laser wavelength and obtained under this new excitation condition, similar spectra with the same temporal behaviour (Figure 4).

\section{DISCUSSION}

From our results, it appears clearly that in inert solvents our results perfectly match the Harris results and interpretation, taking into account, in our case, a different time resolution. Increasing the donor character of the solvent induces deviation from this model, in the sense that new deactivation pathways are opened. Indeed, for the weakly complexing case, new transients appear which come from a competition of molecular $\mathrm{I}_{2}{ }^{*}$-solvent complex relaxation to a lower excited or ground state with the formation of atomic iodine complexes. The absorption spectra of such species appear in the spectral range $680-800 \mathrm{~nm}$ as an additional long-lived transient absorption. In pyridine, a strongly complexing solvent, we did not observe any relaxation process of 

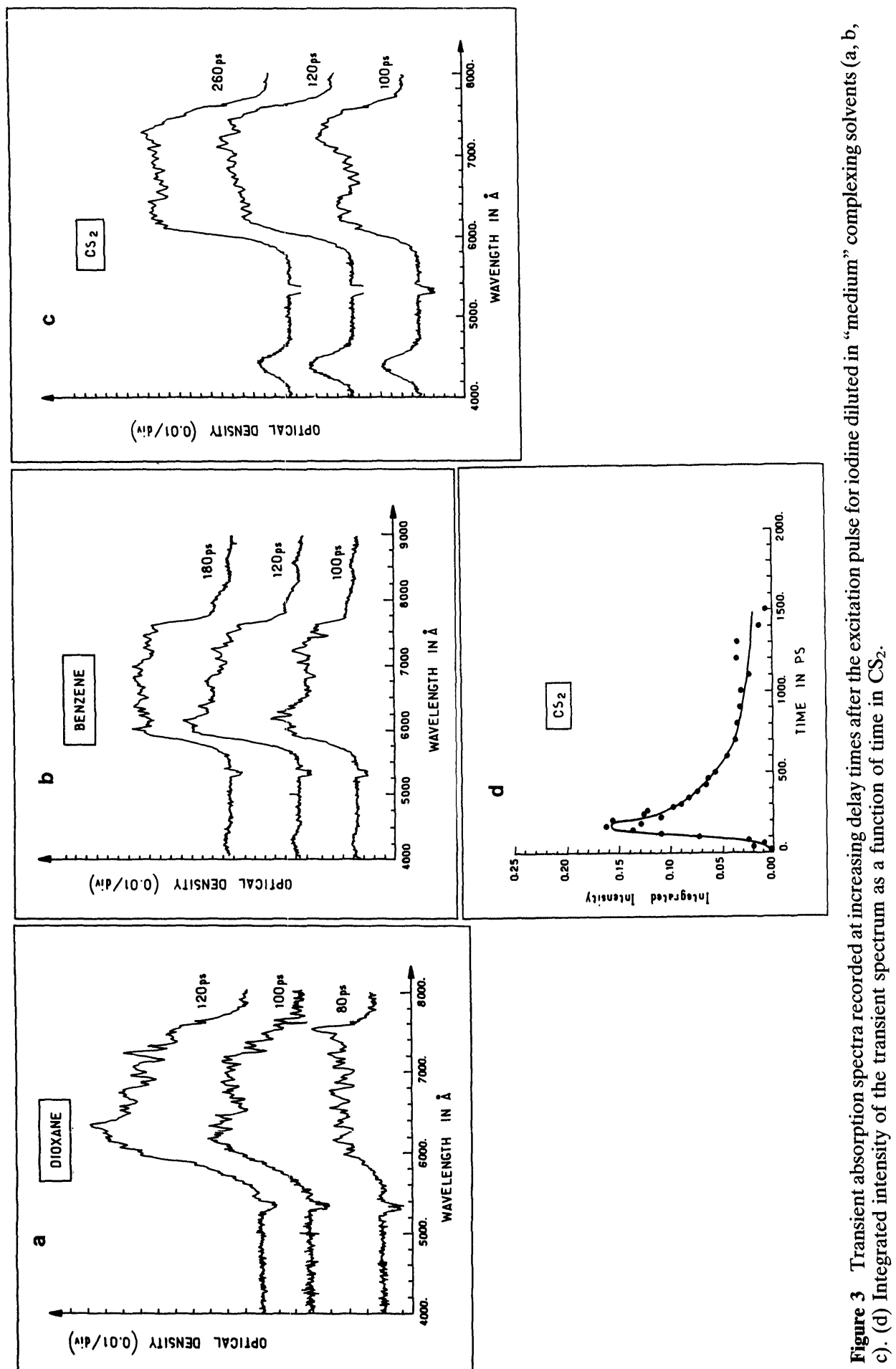

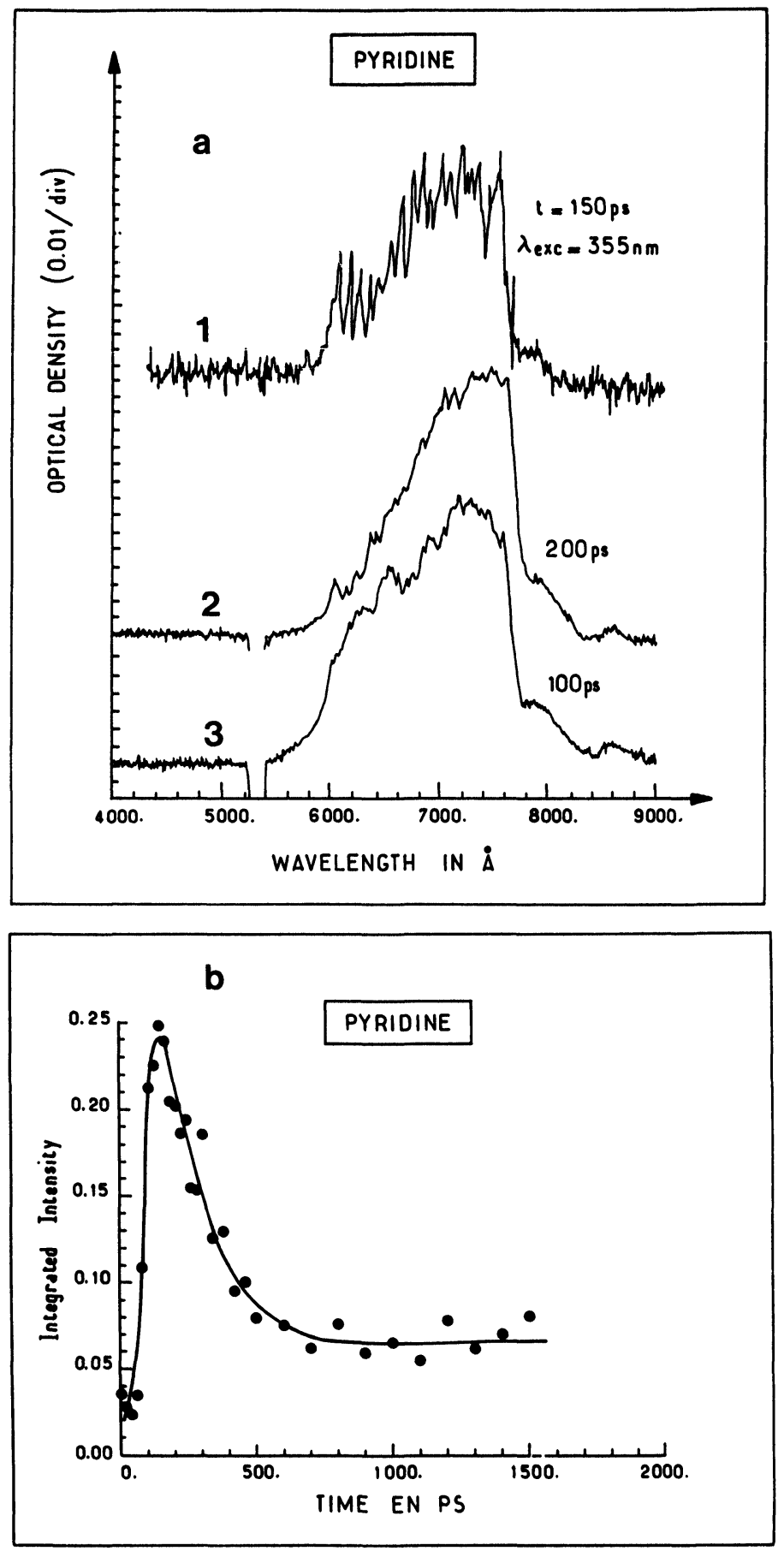

Figure 4 Transient absorption spectra recorded at different times after the excitation in $\mathrm{I}_{2}$-pyridine solution (a) (1) $t=150 \mathrm{ps}, \lambda_{\text {exc }}: 355 \mathrm{~nm}$, (2) $t=200 \mathrm{ps}, \lambda_{\text {exc }}=530 \mathrm{~nm}$ (3) $t=100 \mathrm{ps}, \lambda_{\text {exc }}=530 \mathrm{~nm}$. (b) Integrated intensity of the transient spectra as a function of time. 
the excited molecular iodine complex but only the formation of atomic iodine complex: this shows that complexation does compete efficiently with the vibrational relaxation of excited molecular iodine.

The interpretation of our results may be summarized as follows. In inert solvents, the following reaction occurs:

$$
\mathrm{I}_{2} \stackrel{\text { hy }}{\longrightarrow} \mathrm{I}^{\bullet}+\mathrm{I}^{\bullet} \Rightarrow \mathrm{I}_{2}{ }^{*} \Rightarrow\left\{\begin{array}{l}
\mathrm{I}^{*}{ }_{2 \mathrm{GS}} \\
\mathrm{I}^{*}{ }_{2 \mathrm{~A}, \mathrm{~A}^{\prime}}
\end{array}\right.
$$

The photodissociation of iodine which gives rise to the formation of atomic iodine $\left(\mathrm{I}^{\bullet}\right)$ is followed by geminate recombination which leads to excited molecular iodine $\left(\mathrm{I}_{2}{ }_{2}\right)$.

These two steps are completed in less than 2 ps. After the processes, excited recombined molecular iodine is trapped either in the $\mathrm{A}, \mathrm{A}^{\prime}$ excited electronic states $\left(\mathrm{I}_{2}{ }_{2} \mathrm{~A}, \mathrm{~A}^{\prime}\right)$, the life-time of which are in the range $65-2700 \mathrm{ps}$ or in vibrationally excited levels of the $X$ ground state ( $\mathrm{I}^{*}{ }_{2 \mathrm{GS}}$ ) which relaxes on a $65-150 \mathrm{ps}$ time. This is in agreement with the Harris scheme. ${ }^{1-4}$

In the case of strongly complexing solvents (pyridine), the photodissociation give rise to the following reaction:

$$
\mathrm{I}_{2} \text {-solv } \stackrel{\mathrm{hv}}{\longrightarrow} \mathrm{I}^{\bullet}+\mathrm{I}^{\bullet} \text {-solv }
$$

where $\mathrm{I}_{2}$-solv and $\mathrm{I}^{\bullet}$-solv are complexes which involve, respectively, molecular iodine in its electronic ground state and atomic iodine produced by the photodissociation process, with the solvent molecules.

Due to the strong complexing character of the solvent, atomic iodine $\mathrm{I}^{\bullet}$ mainly recombine with solvent molecules rather than with $\mathrm{I}^{\bullet}$-solv complex or others $\mathrm{I}^{\bullet}$ iodine atoms according to

$$
\text { solv }+\mathrm{I}^{\bullet}+\mathrm{I}^{\bullet} \text {-solv } \rightarrow 2 \mathrm{I}^{\bullet} \text {-solv }
$$

Two $\mathrm{I}^{\bullet}$-solv complexes are thus formed and correspond to the transient absorption spectra observed in pyridine on a $1 \mathrm{~ns}$ scale. We emphasize again that absorption spectra of this complex are completely different from the ones observed in inert solvent. In particular we do not observe (on the time scale probed in our experiment) any spectral evolution as a function of time.

For weakly complexing solvents, the situation is slightly more complicated because after the photodissociation process two reactive channels may be opened, the quantum yield of which depends upon the donor strength of the solvent. The first one leads to the same reaction as II and III. The second one is:

$$
\mathrm{I}^{\bullet}+\mathrm{I}^{\bullet} \text {-sov } \rightarrow \mathrm{I}^{*}{ }_{2} \text {-solv } \rightarrow\left\{\begin{array}{l}
\mathrm{I}^{*}{ }_{2 \mathrm{GS}} \text {-solv } \\
\mathrm{I}^{*}{ }_{2 \mathrm{~A}}, \mathrm{~A}^{\prime-\text { solv }}
\end{array}\right.
$$


Atomic iodine $\mathrm{I}^{\bullet}$ recombines with geminate $\mathrm{I}^{\bullet}$-solv complexes, giving rise to $I^{*}{ }_{2}$-solv excited complex. The competition between processes III and IV is controlled by the strength of the donor character of the solvent. In the case where two transient species (namely $\mathrm{I}^{\bullet}$-solv and $\mathrm{I}^{*}{ }_{2}$-solv complexes) are present in the solution just after the photodissociation-recombination processes, $\mathrm{I}_{2}{ }_{2}$-solv relaxes in the same way as $\mathrm{I}_{2}{ }_{2}$ in inert solvent. $\mathrm{I}^{*}{ }_{2}$-solv relaxation gives rise to the corresponding population of $\mathrm{A}, \mathrm{A}^{\prime}$ and $X$ ground state of the $\mathrm{I}_{2}{ }^{*}$-solv complex.

We note that the transient spectra corresponding to $I^{*}{ }_{2}$-solv species in the ground and $\mathrm{A}, \mathrm{A}^{\prime}$ states are slightly shifted to higher energy with respect to $\mathrm{I}_{2}^{*}$ species in inert solvent (particularly marked in $\mathrm{CS}_{2}$ and benzene where the shifts are of the order of $100 \AA$ ). This is due to solvent perturbation on the energy level of the $I_{2}$-solv species with respect to $I_{2}$ species.

The presence of I-solv complex is also illustrated by transient spectra obtained at longer times $(t>150 \mathrm{ps})$. These spectra are different from the ones obtained in an inert solvent, since an additional shoulder is observed on the low energy side. This additional shoulder appears in the same wavelength range as the transient spectra observed in pyridine, and is attributed to $\mathrm{I}^{\bullet}$-solv complex absorption spectra. This is a good indication that in intermediate solvent $\mathrm{I}^{\bullet}$-solv complexes are present, together with $\mathrm{I}_{2}{ }_{2}$-solv ones.

Incidentally, we note changes of the ground-state absorption spectra of $\mathrm{I}_{2}$ solutions as a function of the solvent. ${ }^{11}$ When exciting at $532 \mathrm{~nm}$ as (we did here) states other than the $B$ state may be populated and other reactions not taken into account in this paper may occur. However in pyridine, using the third harmonic of the laser $(\lambda=355 \mathrm{~nm})$, similar spectra and temporal features were obtained, showing that our interpretation holds in this case. In fact, it is essential to use an additional excitation wavelength to clarify this situation.

\section{CONCLUSION}

The aim of this work was to obtain information about the photophysical behaviour of molecular iodine diluted in complexing solvents. Our results indicate that in such complexing solvents, the Harris model, originally proposed for $\mathrm{I}_{2}$ diluted in inert solvent, is still valid. But one also needs to consider a competition between geminate recombination of iodine atoms and the complexation with the solvent molecules of the cage. This last process may compete efficiently in strongly complexing solvents.

Finally, it is noteworthy that the interpretation of our experiments actually relies on the knowledge of electronic excited state potential functions as obtained for the isolated (gaseous) or very slightly perturbed iodine molecules (i.e. diluted in "inert solvent").

Therefore, our interpretation might be questioned because we lack the knowledge of such excited state potential functions when $I_{2}$ is diluted in strongly interacting solvent. Such questions should be elucidated and, for that purpose, experiments using picosecond time resolved Raman techniques should be considered. 


\section{Acknowledgments}

Authors are pleased to acknowledge Dr. C. Sourisseau (University of Bordeaux I) and Pr. J. Yarwood (Durham University) for discussions and critically reading the manuscript.

\section{References}

1. A. L. Harris, M. Berg and C. B. Harris, J. Chem. Phys. 84, 788 (1986).

2. M. E. Paige, D. J. Russell and C. B. Harris, J. Chem. Phys. 85, 3699 (1986).

3. D. E. Smith and C. B. Harris, J. Chem. Phys. 87, 2709 (1987).

4. J. K. Brown, C. B. Harris and J. C. Tully, J. Chem. Phys. 89, 6687 (1988).

5. Ph. Bado, C. Dupuy, D. Magde and K. R. Wilson, J. Chem. Phys. 80, 5531 (1984).

6. D. F. Kelly, N. A. Abul-Haj and D. J. Jang, J. Chem. Phys. 80, 4105 (1984).

7. D. J. Nesbitt and J. T. Hynes, J. Chem. Phys. 77, 2130 (1982).

8. D. F. Kelley and P. M. Rentzepis, Chem. Phys. Lett. 85, 85 (1982).

9. C. A. Langhoff, K. Gnådig and K. B. Eisenthal, Chem. Phys. 46, 117 (1980).

10. T. J. Chuang, G. W. Hoffman and K. B. Eisenthal, Chem. Phys. Lett. 25, 201 (1974).

11. M. Tamres and J. Yarwood, in "Spectroscopy and structure of molecular complexes", J. Yarwood (ed.), (Plenum Press, New York, 1973) pp. 217-301.

12. C. Rulliere, A. Declemy and Ph. Lee, Rev. Phys. Appl. 18, 347 (1983). 